\title{
Efficacy of Magnetic Resonance Imaging in BIRADS 3, 4 and 5 Patients Detected on Full Field Digital Mammography: Our Experience
}

\author{
Atul Patil*, Samadhan Pawar, Raj Nagarkar, Bhargav Gaikwad \\ HCG Manavata Cancer Centre, Nashik, India \\ Email: ^academics@manavatacancercentre.com
}

How to cite this paper: Patil, A., Pawar, S. Nagarkar, R. and Gaikwad, B. (2019) Efficacy of Magnetic Resonance Imaging in BIRADS 3, 4 and 5 Patients Detected on Full Field Digital Mammography: Our Experience. Open Journal of Clinical Diagnostics, 9, 33-49.

https://doi.org/10.4236/ojcd.2019.91003

Received: January 8, 2019

Accepted: March 22, 2019

Published: March 25, 2019

Copyright $\odot 2019$ by author(s) and Scientific Research Publishing Inc. This work is licensed under the Creative Commons Attribution-NonCommercial International License (CC BY-NC 4.0). http://creativecommons.org/licenses/by-nc/4.0/ (c) (i) (8) Open Access

\begin{abstract}
Aim of the study: To perform Dynamic contrast enhanced MRI of breast in patients with positive findings (BIRADS 3, 4 and 5) detected on screening mammography; to correlate the findings of digital mammography and contrast enhanced MRI of breast with histopathological examinations. Settings and Design: A prospective observation study was conducted at a single centre, i.e. HCG Manavata Cancer Centre. Materials and Methods: Screening mammography was performed on patients with age $>40$ years and on patients with age 35 - 40 years having positive family history. The positive mammography was reported and the lesions classified according to BIRADS criteria for mammography. Results: Mammographic examination of the breast lesions yielded an overall sensitivity of $97.67 \%$ and a specificity of $85.71 \%$. In our study we combined both morphologic and dynamic parameters and its modification into BIRADS category for lesion classification. The sensitivity of MRI examinations was $97.67 \%$ while the specificity was $71.43 \%$. Spiculated margins were encountered only in malignant lesions $(p=0.0006)$. Statistical correlation was obtained between the pathologically proven benign and malignant lesions regarding their enhancement pattern with $p$ value of $<0.001$. Conclusion: As per the results, dynamic contrast MRI had high sensitivity but limited specificity. We did not find any significant difference between FFDM and MRI in terms of diagnostic accuracy. The use of DWI showed high specificity at cut off point of ADC value $-0.85 \mathrm{~mm}^{2} / \mathrm{s}$. Thus, DWI can be used in addition of morphological and dynamic kinetic characteristics to increase specificity of MRI.
\end{abstract}

\section{Keywords}

Breast Cancer, Screening, Mammography, Magnetic Resonance Imaging 


\section{Introduction}

Breast cancer has become a serious health issue among developing countries. The disease was once considered as a problem of the rich and affluent countries. However, breast cancer has now been rooted in developing countries such as India $[1]$

Breast cancer is the most common form of cancer that affects women in India. As per Breast Cancer India, one woman is diagnosed with breast cancer every four minutes in India. One woman dies due to breast cancer every eight minutes in India [2]. In 2012, an estimated 70,218 women have died of breast cancer which is the highest in the world for that year. The number of women to be diagnosed with breast cancer is expected to increase considering the predominant young population. India has witnessed a remarkable rise in breast cancer cases in the age group of 30 to 50 [2]. The same is likely to increase over the years.

The high mortality rate among women in India due to breast cancer is attributed to the diagnosis of the disease at advanced stages. High breast cancer mortality is associated with low levels of awareness, unorganized referral pathways to diagnosis, limited access to effective treatment at local level and incomplete treatment regimens [3].

Some of the key barriers associated with associated with "low cancer awareness" or "scarcity of awareness" among women include fear, stigma, gender inequity, and reduced engagement in overall screening behaviours [4].

There is no systematic, organized, or government funded screening programs for breast cancer in India. The process for screening in developing countries is based on "opportunistic screening". As per the World Health Organization (WHO), mammography screening is recommended every $1-2$ years for women aged between 50 - 69 years [5]. Mammography is considered as the only system validated for screening with a benefit for early detection (1.5 to 4 years). However, the sensitivity of mammography decreases with increasing breast density [6]. Clinicians look for any mass, calcification, or architectural distortion in mammography screens and then give BIRADS (Breast imaging reporting and data system) score. Screen film mammography was initially performed in India but full-field digital mammography (FFDM) has replaced the former for screening purposes [7].

In breast cancer, angiogenesis is a requirement of neoplastic growth, progression, and metastasis. Contrast-enhanced breast magnetic resonance imaging (MRI) is an ideal imaging modality that helps in representing tumour angiogenesis. In MRI, the contrast enhanced pattern of the tumour correlates to the micro-vessels density which is a characteristic of tumour angiogenesis [8]. The features of dynamic contrast-enhanced MRI (DCE-MRI) such as washout curve type, peripheral rim enhancement, and faster enhancement are correlated with overall survival and recurrence of breast cancer patients [8].

\section{Methods}

A prospective observation study was conducted at a single centre, i.e. HCG 
Manavata Cancer Centre. The inclusion criteria of the study was as follows: 1) Women with a positive BIRADS score (BIRADS 3, 4, and 5); 2) Patients who underwent a breast conservation surgery and a positive mammographic finding (BIRADS 3, 4, and 5). The exclusion criteria of the study was as follows: 1) Women aged 40 years or below exception of women up to 35 years with a positive family history for breast cancer; 2) Patient's with an open breast wound; 3) Pregnant and lactating women; 4) Patients with implants such as pacemaker, cochlear implant, or any metallic prosthesis that affects examination.

\section{Imaging Protocol for the Study}

\subsection{Protocol for FFDM}

Mammography images are obtained in 2 views-Medio lateral oblique (MLO) and Cranio caudal (CC). We use the GE Alfa ST for mammography at our centre.

\subsection{Protocol for Breast MR Imaging}

Standard dynamic contrast-enhanced subtracted breast MRI of both entire breasts was performed using Signa Explorer 1.5 T MRI scanner. All patients were imaged in the prone position in a dedicated double breast coil.

Pulse sequences taken:

A transverse T1-weighted spin-echo sequence was performed for localization purposes. Sagittal fat-suppressed T2-weighted fast spin-echo sequence with the following parameters: TR/TE, 8082/56; field of view 350; slice thickness, $3 \mathrm{~mm}$ with gap of $10 \%$.

A three-dimensional axial fat suppressed T1-weighted fast gradient-recalled echo sequence was obtained before and 4 sets of images (1, 2, 6 and 7 minutes after a bolus injection of $0.2 \mathrm{mmol} / \mathrm{kg}$ of Omniscan.

The following parameters were used: TR/TE 4.1/1.2, field of view $360,34 \mathrm{~cm}$; matrix size; slice thickness $1 \mathrm{~mm}$ with no gap.

Fat suppression and subtraction of pre-contrast from first set of post contrast images was done. Bilateral imaging was done for all sequences.

Morphologic analysis was done on post processed subtracted images. Detailed morphologic analysis was done using MRI BI-RADS Lexicon proposed by American college of radiology11 Lesion types.

- Focus;

- Mass;

- Nonmass.

1) Mass

Shape-Round/Oval/Irregular;

Margin-Circumscribed/Noncircumscribed (Irregular/Spiculated);

Internal Enhancement Characteristics-Homogeneous/Heterogeneous/Rim/Dark Internal Septations.

2) Nonmass 
Distribution-Focal, Linear, Segmental, Regional, Multiple regions, Diffuse;

Internal Enhancement Pattern in Nonmass-Homogeneous, Heterogeneous, Clumped, Clustered Ring;

Kinetic analysis of time signal intensity curve was done for each lesion;

Progressive (Type1)/Plateau (Type 2)/Washout (Type 3).

We used Computer Aided Detection System for visual kinetic assessment of breast lesions.

BIRADS 1, 2, 3 (given by FFDM and MRI) were taken as negative for malignancy and BIRADS 4, 5 (given by FFDM and MRI) lesions were taken as positive for malignancy for calculating sensitivity, specificity, Positive Predictive Value, Negative Predictive Value and, Diagnostic accuracy as compared to HPE. Sensitivity and specificity of DWI was also calculated using mean ADC values for each lesion at b-value of $1500 \mathrm{~s} / \mathrm{mm}^{2}$.

\subsection{Ethical Considerations}

The present study will be conducted in adult patients after written informed consent. The defined guidelines of Central Ethics Committee for Biomedical Research on human subjects by ICMR will be adhered, in addition to the principles enunciated in the Declaration of Helsinki12.

All safety measures and precautions pertaining to patient and health workers will be taken and patients will be given the right to opt out of the study at any time they want to, with no compromise on their health.

Proper counselling and treatment options will be provided to the patients with breast lesions depending on their imaging and histopathology findings.

The hospital is well equipped in dealing with all the emergencies arising out of the procedure.

1) To perform Dynamic contrast enhanced MRI of breast in patients with positive findings (BIRADS 3, 4 and 5) detected on screening mammography.

2) To correlate the findings of digital mammography and contrast enhanced MRI of breast with histopathological examinations.

3) To evaluate sensitivity, specificity, positive predictive value, negative predictive value and accuracy of FFDM and contrast enhanced MRI

4) To evaluate role of DWI in increasing specificity of contrast enhanced MRI

\section{Results}

A total of 50 volunteers were included in the study. The age-wise distribution of study participants are described in Table 1 and Table 2.

\subsection{Mass Morphologic Descriptions}

As we encountered 49 masses and 1 non-mass like enhancement, we calculated morphological and kinetic analysis for 49 masses as described below (Figures $1-8)$.

$p$-value is calculated by fisher's Exact Test via SPSS version 2. 


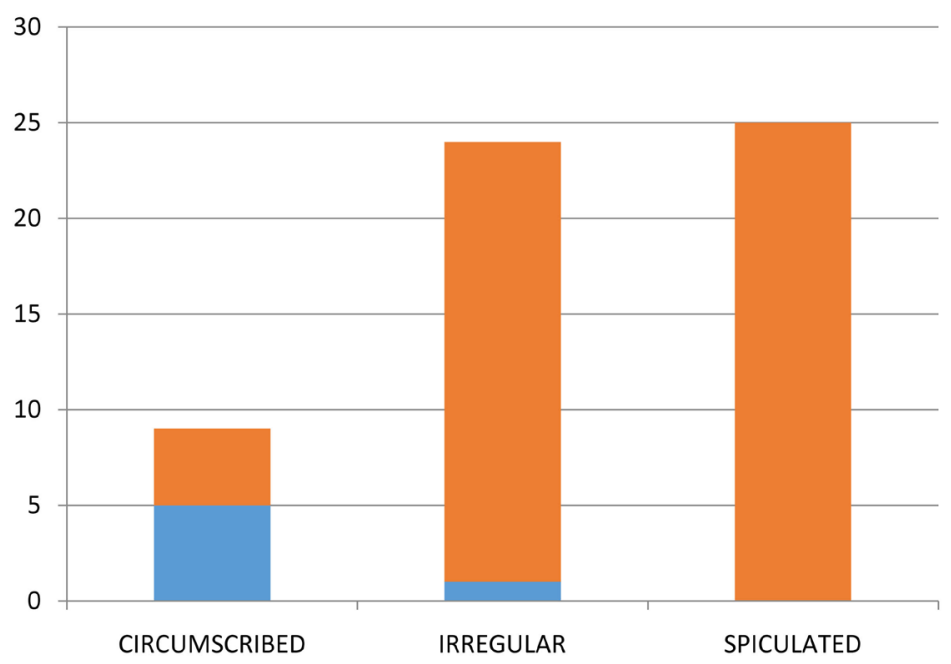

- Column1 MALIGNANT

BENIGN

Figure 1. Mass morphology.

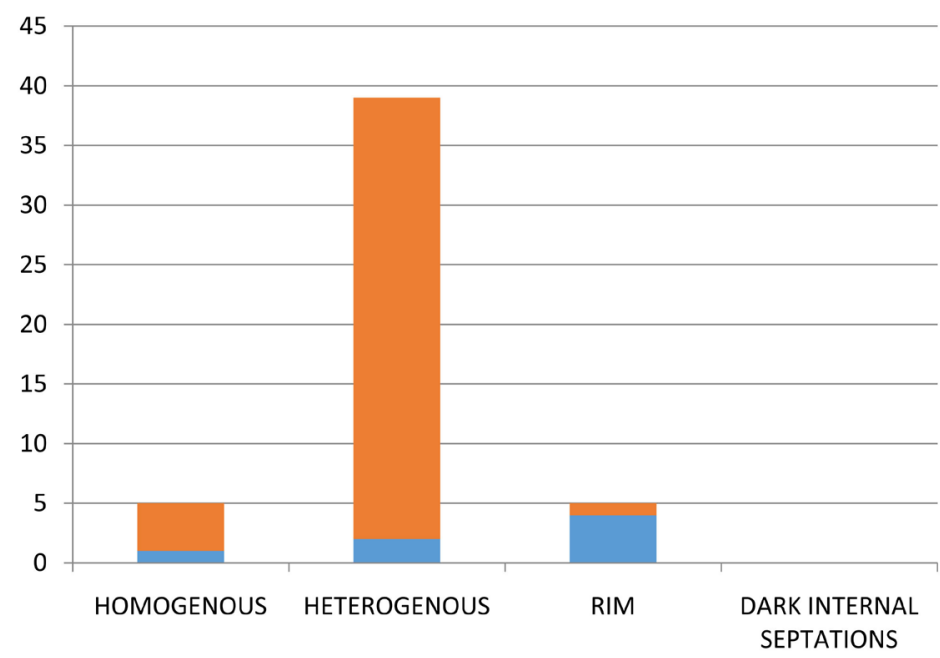

Figure 2. Internal enhancement characteristics.

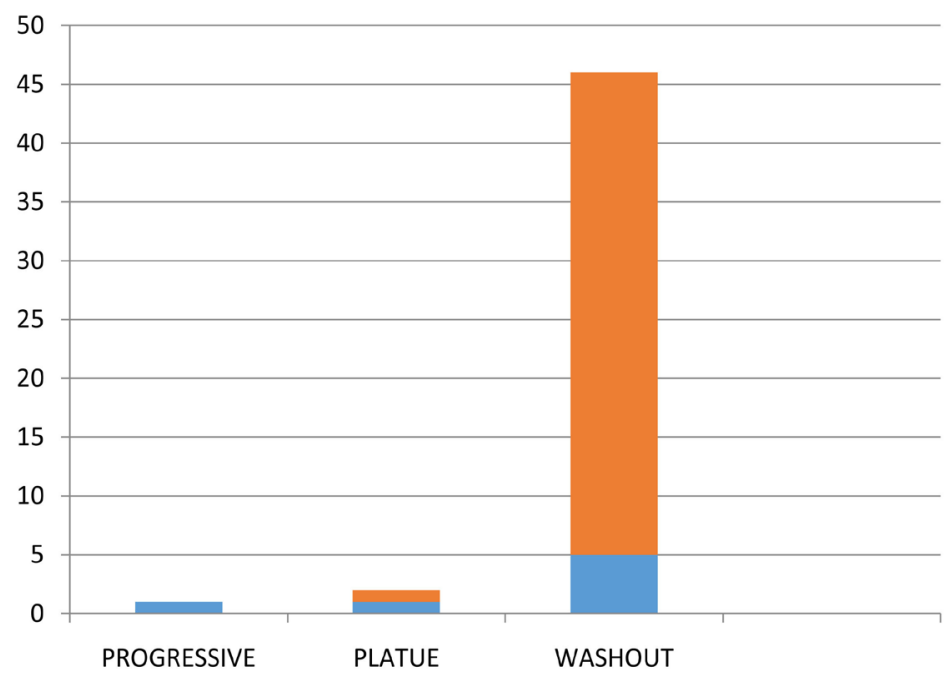

MALIGNANT - BENIGN

Figure 3. Frequency of visually assessed kinetic patterns. 


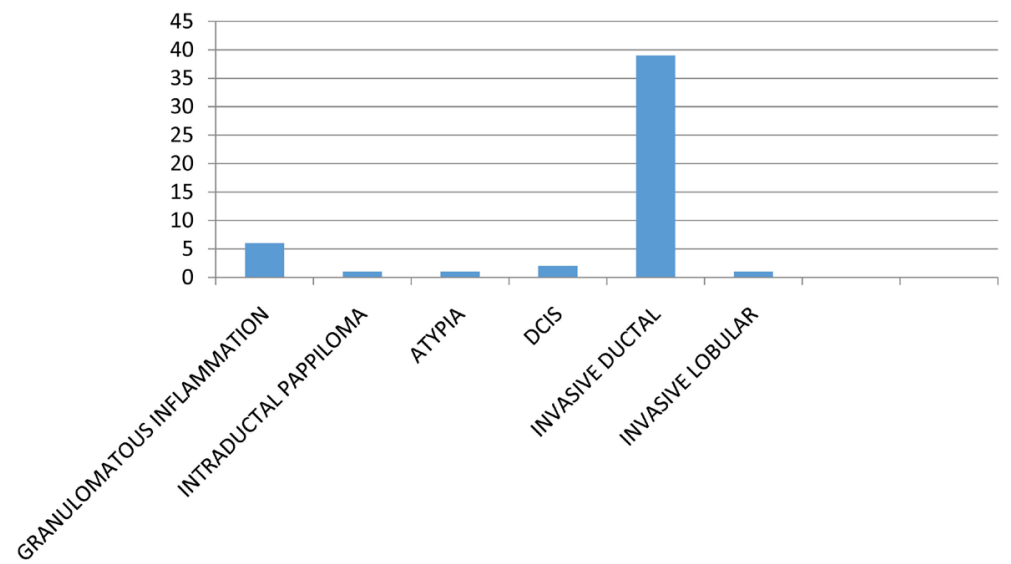

Figure 4. Histopathology of primary lesion.

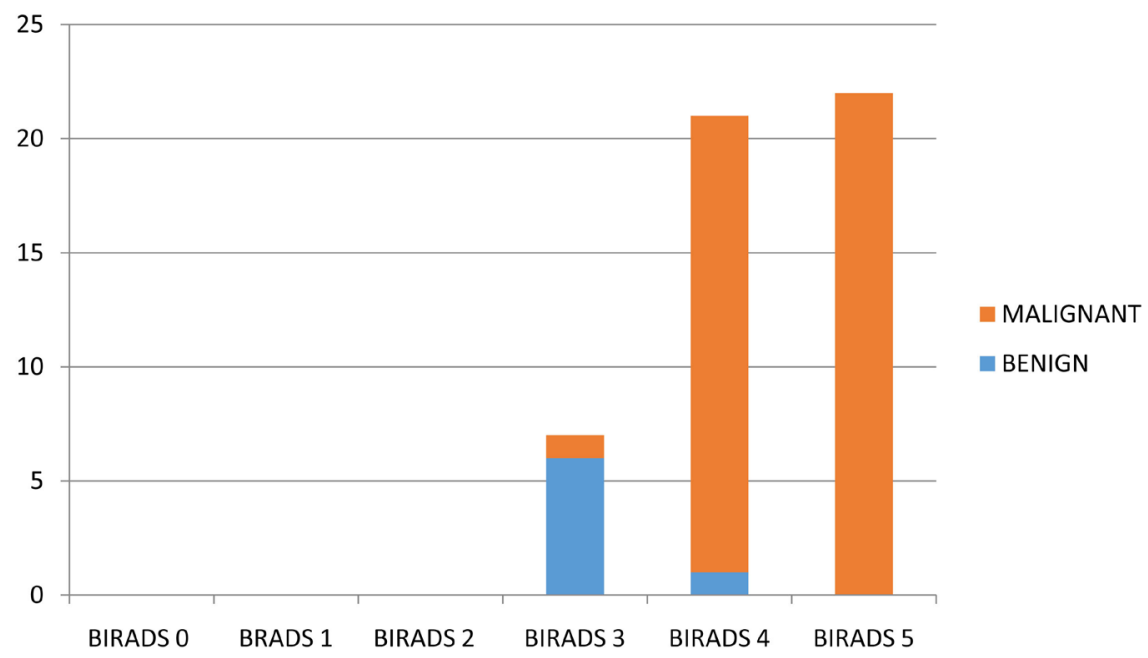

Figure 5. Mammography findings.

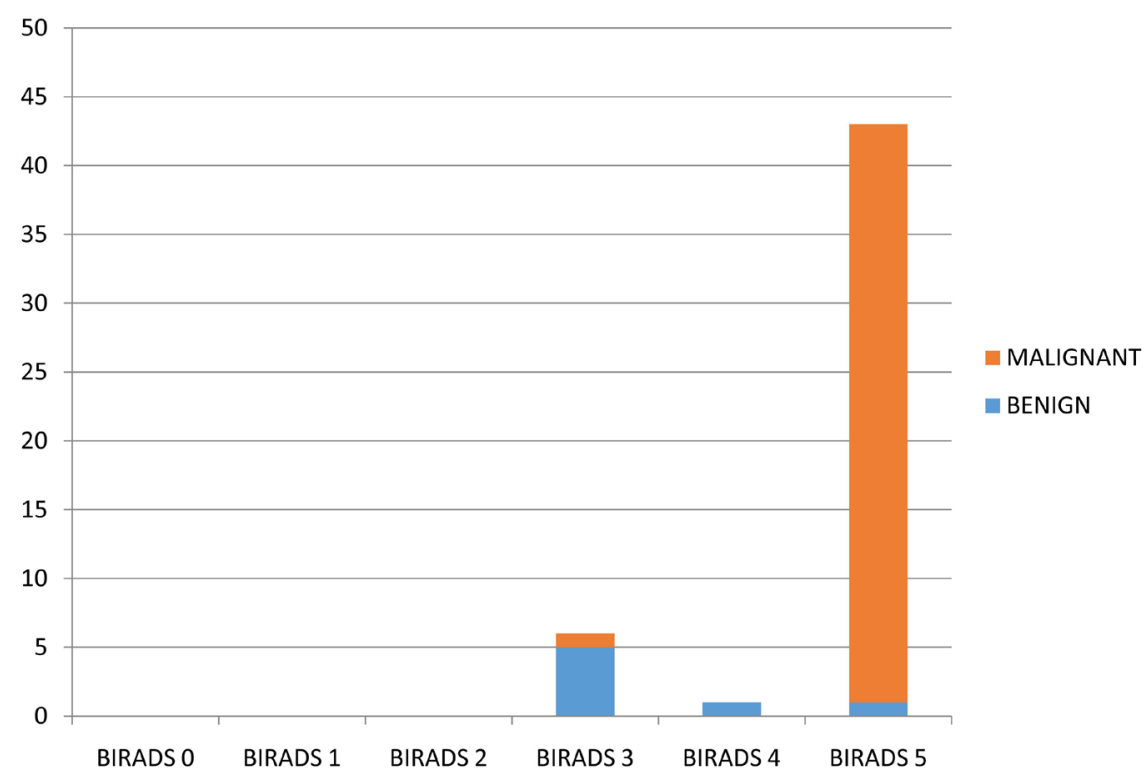

Figure 6. MRI findings. 


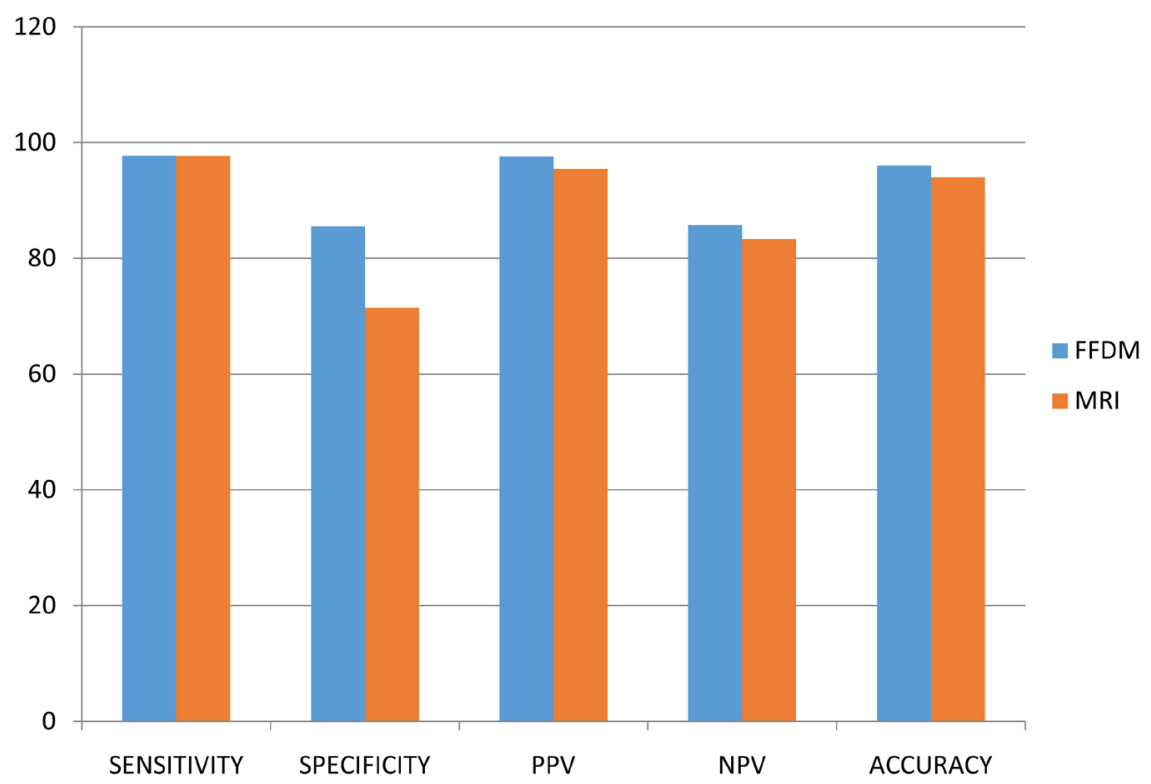

Figure 7. Sensitivity, specificity, PPV, NPV, accuracy FFDM versus MRI.

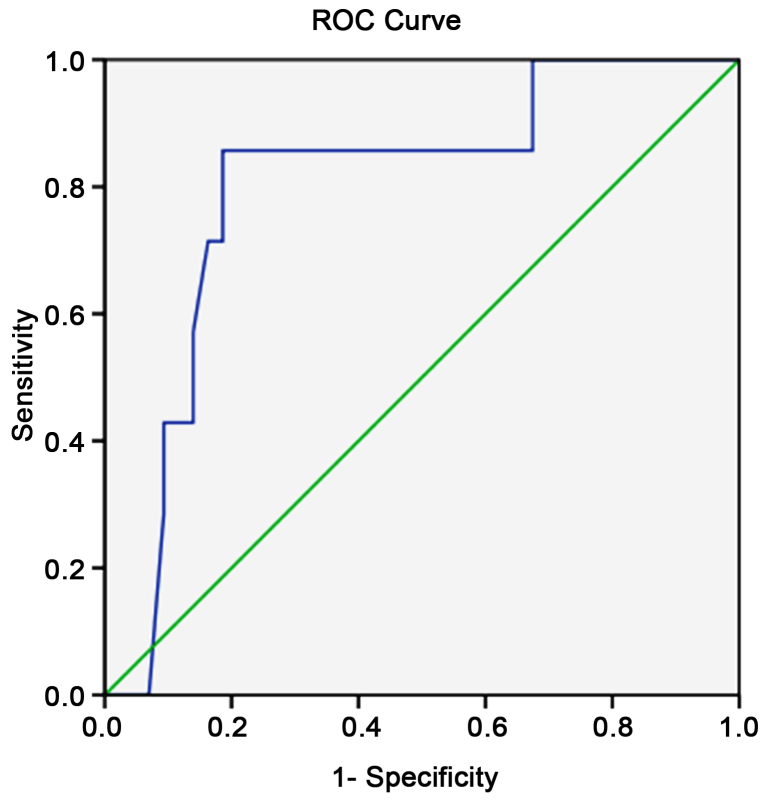

Diagonal segments are produced by ties.

Figure 8. Area under the curve.

Test Result Variable(s): ADC

\begin{tabular}{ccccc}
\hline \multirow{2}{*}{ Area } & Std. Error & Asymptotic Sig. & \multicolumn{2}{c}{ Asymptotic 95\% Confidence Interval } \\
\cline { 4 - 5 } & & & Lower Bound & Upper Bound \\
\hline 0.799 & 0.086 & 0.012 & 0.630 & 0.968 \\
\hline
\end{tabular}

The test result variable(s): $\mathrm{ADC}$ has at least one tie between the positive actual state group and the negative actual state group. Statistics may be biased. ${ }^{a}$ Under the nonparametric assumption; ${ }^{b}$ Null hypothesis: true area $=0.5$. 
Table 1. Age wise distribution.

\begin{tabular}{cc}
\hline Age & Number of patients \\
\hline $35-39$ & 10 \\
$40-44$ & 7 \\
$45-49$ & 7 \\
$50-54$ & 9 \\
$55-59$ & 6 \\
$60-64$ & 9 \\
$>65$ & 2 \\
\hline
\end{tabular}

Table 2. MRI description of lesions using BI-RADS lexicon.

\begin{tabular}{cc}
\hline Type of lesion & Number of lesions \\
\hline Focus & 0 \\
Mass & 49 \\
Nonmass & 1 \\
Total & 50 \\
\hline
\end{tabular}

\section{1) Margin}

Irregular margin observed in 1 benign and 23 malignant lesions. Spiculated margins were observed in only malignant lesions. Circumscribed margins were observed in both benign and malignant lesions (Table 3).

\section{2) Internal enhancement characteristics}

Malignant lesions showed heterogeneous enhancement in 37 lesions, homogenous enhancement in 4 lesions and rim enhancement in 1 lesion. $p$-value for internal enhancement characteristics was significant (Table 4).

\subsection{Frequency of Visually Assessed Kinetic Patterns}

A total of 46 lesions were of type 3 kinetics of which give were benign. Two lesions were of type 2 kinetics while a single lesion was of type 1 kinetics (Table 5 and Table 6).

\subsection{Mammography}

As our study criteria are evaluations of lesions with positive mammography findings in patients undergoing screening mammography, we have only BIRADS 3, 4, and 5 lesions.

A total of 6 BIRADS 3 lesions were observed of which one was malignant while a total of 21 BIRADS 4 lesions were observed of which one was malignant. All BIRADS 5 lesions were malignant (Table 7).

\subsection{The Sensitivity, Specificity, PPV, NPV, Accuracy of FFDM Was Calculated Using OpenEpi Software}

BIRADS 4 and 5 lesions on FFDM considered as positive and BIRDAS 3 on FFDM as negative for malignancy (Table 8). 
Table 3. Mass morphologic descriptions.

\begin{tabular}{ccccc}
\hline Margin & Benign & Malignant & Total & $p$ value \\
\hline Circumscribed (smooth) & 5 & 4 & 9 & \\
Noncircumscribed (irregular) & 1 & 23 & 24 & $p=0.006$ \\
Noncircumscribed (spiculated) & 0 & 15 & 16 & \\
Total & 7 & 42 & 49 & \\
\hline
\end{tabular}

p-value for margin was significant.

Table 4. Internal enhancement characteristics.

\begin{tabular}{ccccc}
\hline Internal enhancement & Benign & Malignant & Total & $p$ value \\
\hline Homogenous & 1 & 4 & 5 & \\
Heterogeneous & 2 & 37 & 39 & $p<0.001$ \\
Rim & 4 & 1 & 5 & \\
Dark internal septation & 0 & 0 & 49 & \\
Total & 0 & 0 & &
\end{tabular}

Table 5. Frequency of visually assessed kinetic patterns.

\begin{tabular}{ccccc}
\hline Type of curve & Benign & Malignant & Total & $p$ value \\
\hline Washout & 5 & 41 & 46 & \\
Platue & 1 & 1 & 2 & $p=0.050$ \\
Progressive & 1 & 0 & 1 & \\
Total & 7 & 43 & 49 & \\
\hline
\end{tabular}

p-value in this case was insignificant.

Table 6. Histopathology of primary lesions.

\begin{tabular}{cc}
\hline Type of lesion & Number of patients \\
\hline BENIGN & 73 \\
\hline MALIGNANT & Number of patients \\
Histology & 7 \\
Benign & 0 \\
Precancerous & 1 \\
Atypia & 0 \\
Malignant & 39 \\
Invasive ductal & 1 \\
Invasive lobular & 2 \\
Insitu ductal & 0 \\
Medullary & 0 \\
Tubular & \\
\hline
\end{tabular}


Table 7. Mammography findings.

\begin{tabular}{cccccccc}
\hline \multirow{2}{*}{ Histopathology } & \multicolumn{7}{c}{ Mammography Birads } \\
\cline { 2 - 8 } & $\mathbf{0}$ & $\mathbf{1}$ & $\mathbf{2}$ & $\mathbf{3}$ & $\mathbf{4}$ & $\mathbf{5}$ & Total \\
\hline Benign & 0 & 0 & 0 & 6 & 1 & 0 & 7 \\
Malignant & 0 & 0 & 0 & 1 & 20 & 22 & 43 \\
Total & 0 & 0 & 0 & 7 & 21 & 22 & 50 \\
\hline
\end{tabular}

Table 8. Sensitivity, specificity, PPV, NPV, ACCURACY of FFDM.

\begin{tabular}{cccc}
\hline Parameter & Estimate & Lower - Upper 95\% CIs & Method \\
\hline Sensitivity & $97.67 \%$ & $(87.94,99.591)$ & Wilson Score \\
Specificity & $85.71 \%$ & $(4.8 .69,97.431)$ & Wilson Score \\
Positive Predictive Value & $97.67 \%$ & $(87.94,99.591)$ & Wilson Score \\
Negative Predictive Value & $85.71 \%$ & $(48.69,97.431)$ & Wilson Score \\
Diagnostic Accuracy & $96 \%$ & $(86.54,98.91)$ & Wilson Score \\
Likelihood ratio of a Positive Test & 6.837 & $(0.962-48.59)$ & \\
Likelihood ratio of a Negative Test & 0.02713 & $(0.003619-0.2034)$ & \\
Diagnostic Odds & 252 & $(13.85-4584)$ & \\
Cohen's kappa (Unweighted) & 0.8339 & $(0.5567-1.111)$ & \\
Entropy reduction after a Positive Test & $29.45 \%$ & & \\
Entropy reduction after a Negative Test & $-0.5153 \%$ & & \\
Bias Index & 0.0 & & \\
\hline
\end{tabular}

\subsection{MRI}

MRI classified 6 patients as BIRADS 3 out of which 1 turned out as malignant. A single patient as BIRADS 4 which turned out as benign and 43 patients as BIRADS 5 out of which 1 turned out as benign (Table 9).

The Sensitivity, Specificity, PPV, NPV, Accuracy of MRI was calculated using OpenEpi software. BIRADS 4 and 5on MRI considered as positive and BIRDAS 3 on MRI as negative for Malignancy (Table 10 and Table 11).

\subsection{Evaluation of DWI Is Calculated Using WINPEPI Software}

Area under the ROC curve $=79.9 \%$ (S.E. $=8.64 \%$ );

$95 \%$ confidence interval $=63.0 \%$ to $96.8 \%$;

Significance [compared with $50 \%^{\star}$ ]: $p=0.000$ [2.7E-4];

Confidence band not shown in graph (small numbers) (Table 12).

Sensitivity of DWI-81.4\% and Specificity of DWI-85.7\% observed to be maximum at cut off ADC value of $0.856 \mathrm{~mm}^{2} / \mathrm{s}$.

\section{Discussion}

Breast MRI is gaining increasing clinical acceptance in multiple applications, 
Table 9. MRI findings.

\begin{tabular}{cccccccc}
\hline \multirow{2}{*}{ Histopathology } & \multicolumn{7}{c}{ MRI Birads } \\
\cline { 2 - 8 } & $\mathbf{0}$ & $\mathbf{1}$ & $\mathbf{2}$ & $\mathbf{3}$ & $\mathbf{4}$ & $\mathbf{5}$ & Total \\
\hline Benign & 0 & 0 & 0 & 5 & 1 & 1 & 7 \\
Malignant & 0 & 0 & 0 & 1 & 0 & 42 & 43 \\
Total & 0 & 0 & 0 & 6 & 1 & 43 & 50 \\
\hline
\end{tabular}

Table 10. The sensitivity, specificity, PPV, NPV, accuracy of MRI.

\begin{tabular}{cccc}
\hline Parameter & Estimate & Lower - Upper 95\% CIs & Method \\
\hline Sensitivity & $97.67 \%$ & $(87.94,99.591)$ & Wilson Score \\
Specificity & $71.43 \%$ & $(35.89,91.781)$ & Wilson Score \\
Positive Predictive Value & $95.45 \%$ & $(84.86,98.741)$ & Wilson Score \\
Negative Predictive Value & $83.33 \%$ & $(43.65,96.991)$ & Wilson Score \\
Diagnostic Accuracy & $94 \%$ & $(83.78,97.941)$ & Wilson Score \\
Likelihood ratio of a Positive Test & 3.419 & $(1.282-9.119)$ & \\
Likelihood ratio of a Negative Test & 0.03256 & $(0.003921-0.2704)$ & \\
Diagnostic Odds & 105 & $(8.009-1377)$ & \\
Cohen's kappa (Unweighted) & 0.735 & $(0.4589-1.011)$ & \\
Entropy reduction after a Positive Test & $22.01 \%$ & & \\
Entropy reduction after a Negative Test & $-4.56 \%$ & & \\
Bias Index & 0.02 & & \\
\hline
\end{tabular}

Table 11. Sensitivity, specificity, PPV, NPV, Accuracy FFDM versus MRI.

\begin{tabular}{ccc}
\hline & FFDM & Contrast MRI \\
\hline Sensitivity & $97.7 \%$ & $97.67 \%$ \\
Specificity & $85.5 \%$ & $71.43 \%$ \\
PPV & $97.6 \%$ & $95.45 \%$ \\
NPV & $85.7 \%$ & $83.33 \%$ \\
Accuracy & $96 \%$ & $94 \%$
\end{tabular}

Table 12. Dichotomizing the results as "negative" or positive" using different cut points (defining results at or below the cut point as "positive").

\begin{tabular}{ccccc}
\hline Cut-point & Sensitivity (\%) & Specificity (\%) & Diagnostic OR & Youden's index (\%) \\
\hline 0.513 & 2.3 & 100.0 & inf. & 2.3 \\
0.52 & 4.7 & 100.0 & inf. & 4.7 \\
0.525 & 7.0 & 100.0 & inf. & 7.0 \\
0.528 & 9.3 & 100.0 & inf. & 9.3 \\
0.539 & 11.6 & 100.0 & inf. & 11.6 \\
0.565 & 14.0 & 100.0 & inf. & 14.0 \\
0.569 & 16.3 & 100.0 & inf. & 16.3 \\
0.572 & 18.6 & 100.0 & inf. & 18.6 \\
\hline
\end{tabular}




\section{Continued}

\begin{tabular}{|c|c|c|c|c|}
\hline 0.589 & 20.9 & 100.0 & inf. & 20.9 \\
\hline 0.604 & 23.3 & 100.0 & inf. & 23.3 \\
\hline 0.616 & 25.6 & 100.0 & inf. & 25.6 \\
\hline 0.622 & 27.9 & 100.0 & inf. & 27.9 \\
\hline 0.627 & 30.2 & 100.0 & inf. & 30.2 \\
\hline 0.629 & 32.6 & 100.0 & inf. & 32.6 \\
\hline 0.633 & 32.6 & 85.7 & 2.9 & 18.3 \\
\hline 0.636 & 34.9 & 85.7 & 3.2 & 20.6 \\
\hline 0.65 & 37.2 & 85.7 & 3.6 & 22.9 \\
\hline 0.671 & 41.9 & 85.7 & 4.3 & 27.6 \\
\hline 0.693 & 44.2 & 85.7 & 4.7 & 29.9 \\
\hline 0.705 & 46.5 & 85.7 & 5.2 & 32.2 \\
\hline 0.707 & 48.8 & 85.7 & 5.7 & 34.6 \\
\hline 0.73 & 51.2 & 85.7 & 6.3 & 36.9 \\
\hline 0.731 & 53.5 & 85.7 & 6.9 & 39.2 \\
\hline 0.752 & 55.8 & 85.7 & 7.6 & 41.5 \\
\hline 0.76 & 58.1 & 85.7 & 8.3 & 43.9 \\
\hline 0.775 & 60.5 & 85.7 & 9.2 & 46.2 \\
\hline 0.78 & 62.8 & 85.7 & 10.1 & 48.5 \\
\hline 0.783 & 65.1 & 85.7 & 11.2 & 50.8 \\
\hline 0.785 & 67.4 & 85.7 & 12.4 & 53.2 \\
\hline 0.808 & 69.8 & 85.7 & 13.8 & 55.5 \\
\hline 0.824 & 72.1 & 85.7 & 15.5 & 57.8 \\
\hline 0.825 & 74.4 & 85.7 & 17.5 & 60.1 \\
\hline 0.827 & 76.7 & 85.7 & 19.8 & 62.5 \\
\hline 0.839 & 79.1 & 85.7 & 22.7 & 64.8 \\
\hline 0.856 & 81.4 & 85.7 & 26.2 & 67.1 \\
\hline 0.864 & 81.4 & 71.4 & 10.9 & 52.8 \\
\hline 0.888 & 83.7 & 71.4 & 12.9 & 55.1 \\
\hline 0.89 & 86.0 & 57.1 & 8.2 & 43.2 \\
\hline 0.987 & 86.0 & 42.9 & 4.6 & 28.9 \\
\hline 1 & 88.4 & 42.9 & 5.7 & 31.2 \\
\hline 1.1 & 90.7 & 42.9 & 7.3 & 33.6 \\
\hline 1.2 & 90.7 & 28.6 & 3.9 & 19.3 \\
\hline 1.22 & 93.0 & 0.0 & 0 & -7.0 \\
\hline 1.296 & 95.3 & 0.0 & 0 & -4.7 \\
\hline 1.402 & 97.7 & 0.0 & 0 & -2.3 \\
\hline
\end{tabular}


including screening, staging biopsy-proven breast cancer and detecting contra lateral disease. The excellent sensitivity of MRI for invasive breast cancer detection is beneficial, but its varying specificity has proved problematic.

The lack of standardized MRI acquisition techniques and image interpretation based on lesion morphology and kinetics has limited clinical implementation at many MRI centers.

We conducted a study of 50 patients to evaluate the role of DCE MRI in characterising the probably benign and suspicious breast findings (BIRADS 3, 4 and 5) after mammographic examinations in terms of sensitivity, specificity, PPV, NPV and diagnostic accuracy as compared to Full field digital mammography and role of DWI in increasing specificity of MRI.

\subsection{Full Field Digital Mammography}

In our study, mammographic examination of the breast lesions yielded an overall sensitivity of $97.67 \%$, a specificity of $85.71 \%$. As per previously published data, the sensitivity of and specificity of mammography was found to be $71.8 \%$ and $97 \%$ respectively [9]. Breast density is a key factor that limits the sensitivity for FFDM or MRI [10]. High sensitivity of mammography in our study may be attributed to selection of only BIRADS 3, 4 and 5 cases unlike studies which evaluated general population and healthy women who undergo periodic screening. Mammography is extremely sensitive in detecting micro calcifications even though it is inadequate for differentiating malignant from benign lesions and invasive from in situ carcinomas [11]. In our study we encountered five lesions with micro calcifications, four of them were given mammographic BIRADS score of 4 and one was given a BIRADS score of 5. The histopathological results yielded 3 cases of invasive ductal carcinoma and two cases of DCIS.

\subsection{Dynamic Contrast MRI}

Breast MRI is a clinically useful additional diagnostic tool and has an excellent sensitivity which usually exceeds $90 \%$. However, the overall specificity of breast MRI varies between $67 \%$ and $72 \%$ [12]. As per ACR BIRADS LEXICON, there are certain morphological and dynamic contrast enhancement characteristics for assessing lesions on MRI. Combinations of different dynamic and morphological characteristics have been reported that can reach diagnostic sensitivities up to $97 \%$ and specificities up to $76.5 \%$ [13]. In our study we combined both morphologic and dynamic parameters and its modification into BIRADS category for lesion classification. The sensitivity of MRI examinations was $97.67 \%$ while the specificity was $71.43 \%$. In general, margin and shape analysis should be performed on first post contrast image to avoid washout and progressive enhancement of the surrounding breast tissue [14].

\subsection{Morphological Characteristics}

Spiculated margins are suspicious for carcinoma, having $91 \%$ positive predictive 
value for malignancy [15]. In our study spiculated margins were encountered only in malignant lesions. Irregular margins were observed in one benign and 23 malignant lesions. Smooth margins were observed in five benign and four malignant lesions. The calculated $p$-value of mass margin in differentiating benign from malignant was 0.006 .

\subsection{Internal Enhancement Characteristics}

Some of the most powerful diagnostic criteria for the differentiation of benign and malignant tumours belong to internal enhancement of focal mass [16]. The attribute of neoangiogenesisis used in malignant lesions which are often too small to be proved by another imaging method [17]. A team of researchers reported that the most frequent morphological finding among the malignant lesions was heterogeneous internal enhancement [18].

In our study 37 malignant lesions and 2 benign lesions exhibited heterogeneous enhancement, its pathological diagnosis was Granulomatous inflammation. Ring enhancement was found in one malignant case. Homogenous enhancement was found in 5 lesions, 1 was benign and 4 were malignant. In our study there was statistical correlation between the pathologically proven benign and malignant lesions regarding their enhancement pattern with $p$-value of $<0.001$, heterogeneous enhancement being encountered more commonly in malignant lesions.

\subsection{Dynamic Contrast Analysis (Kinetic Curve)}

The curve shape is an important differentiator between cancer and benign lesions for comparable enhancement rates. The majority of benign lesions have a Type I and majority of malignant lesions have type III curve. Unfortunately there is overlap within these categories 23.

We calculated the $p$-value of each type of time signal intensity curve and we found that progressive (type I ) and the wash out (type III) curves were found in 1 and 5 pathologically proven benign lesions compared to 0 and 41 malignant lesions respectively. $p$-value was insignificant $(=0.05)$ in differentiation benign from malignant lesions.

\subsection{Role of DWI}

A team of experts reported $94.4 \%$ sensitivity and $84.4 \%$ specificity of DWI in the characterization of lesions with ADC cut off $0.90 \mathrm{~mm}^{2} / \mathrm{s}$ [19]. Rubesova et al. in their study found that the threshold between malignant and benign lesions for the highest sensitivity and specificity (both $86 \%$ ) was around $1.13 \times 10^{-3} \mathrm{~mm}^{2} / \mathrm{s}$ [20]. Similarly, Imamura et al. reported that the most feasible ADC value to depict malignant lesions was found to be less than $1.10 \times 10^{-3} \mathrm{~mm}^{2} / \mathrm{s}$ [21]. Using this threshold sensitivity and specificity were $68.8 \%$ and $72.7 \%$ respectively [21]. Also, Yabuuchi et al. demonstrated an ADC value less than $1.30 \times 10^{-3} \mathrm{~mm}^{2} / \mathrm{s}$ as the strongest indicator of malignancy [22]. In our study, DWI had a sensitivity 
of $81 \%$ and specificity of $85.7 \%$ at ADC cut off value $0.85 \mathrm{~mm}^{2} / \mathrm{s}$. Our findings showed that the best ADC cut off value to differentiate between benign and malignant lesions is $0.85 \mathrm{~mm}^{2} / \mathrm{s}$ at b-value of $1500 \mathrm{~s} / \mathrm{mm}^{2}$. This difference in ADC threshold can be explained by difference in many technical variables that can affect ADC values, such as different MRI units, pulse sequences or b-values [22]. Palle and Reddy found that the ADC value obtained with low b-values (0 - 150 $\mathrm{s} / \mathrm{mm}^{2}$ ) is higher than that obtained with higher b-values (499 and $1500 \mathrm{~s} / \mathrm{mm}^{2}$ ) for all lesion types due to contribution of main perfusion effects to the ADC [23]. Therefore we calculated the ADC value with highest b-values $\left(1500 \mathrm{~s} / \mathrm{mm}^{2}\right)$ to avoid signal attenuation effects at low b-values.

\subsection{Role of MRI in Comparison to FFDM}

All BIRADS 5 lesions on FFDM turned out to be malignant on HPE. There was only 1 of all BIRADS 4 lesions on FFDM which turned out to benign. MRI did not offer any advantage in that case. MRI also showed BIRADS 4 for the same lesion. Only 1 out of all BIRADS 3 lesions on FFDM turned out to be malignant which was intraductal papilloma with DCIS. MRI labelled it as BIRADS 5 lesions. Thus, MRI offered an advantage as we could find one false negative on FFDM.

\section{Conclusion}

Our study showed high sensitivity for Dynamic contrast MRI but with limited specificity. We did not find any significant difference between FFDM and MRI in terms of diagnostic accuracy. MRI did not offer any significant advantage in characterising BIRADS 3, 4 and 5 lesions except upgrading one false negative finding on FFDM. So MRI should not be used in every case of BIRADS 3, 4 and 5 lesions on FFDM. It could be used as a problem solving modality when FFDM and ultrasound shows equivocal findings and biopsy is not feasible but it requires further large scale studies. Morphological descriptors scored over dynamic enhancement characteristics in our study. Morphological descriptors such as margin and internal characteristics showed significant $p$-value with spiculated, irregular margins and heterogeneous internal enhancement of lesions being strong predictors of malignancy. Use of DWI showed high specificity at cut off point of ADC value- $0.85 \mathrm{~mm}^{2} / \mathrm{s}$. Thus, DWI can be used in addition of Morphological and dynamic kinetic characteristics to increase specificity of MRI.

\section{Limitations}

Our study showed high sensitivity for mammography as we included only BIRADS 3, 4 and 5 lesions instead of screening general population. Our study population was limited to 50 and there were only 7 benign cases. Thus, large number of sample size is required to evaluate role of MRI as a problem solving tool. We came across only a single case of non-mass like enhancement, so we 
could not evaluate predictors of malignancy in non-mass like enhancement.

\section{Acknowledgements}

We would like to thank Mr. Lyndon Fernandes for his medical writing assistance.

\section{Conflicts of Interest}

The authors declare no conflicts of interest regarding the publication of this paper.

\section{References}

[1] Singh, S., Shrivastava, J.P. and Dwivedi, A. (2015) Breast Cancer Screening Existence in India: A Nonexisting Reality. Indian Journal of Medical and Paediatric Oncology, 36, 207-209. https://doi.org/10.4103/0971-5851.171539

[2] Breast Cancer India. http://breastcancerindia.net/

[3] Gupta, A., Shridhar, K. and Dhillon, P.K. (2015) A Review of Breast Cancer Awareness among Women in India: Cancer Literate or Awareness Deficit? European Journal of Cancer, 51, 2058-2066. https://doi.org/10.1016/j.ejca.2015.07.008

[4] Dey, S. (2014) Preventing Breast Cancer in LMICs via Screening and/or Early Detection: The Real and the Surreal. World Journal of Clinical Oncology, 5, 509-519. https://doi.org/10.5306/wjco.v5.i3.509

[5] Takkar, N., Kochhar, S., Garg, P., Pandey, A.K., Dalal, U.R. and Handa, U. (2017) Screening Methods (Clinical Breast Examination and Mammography) to Detect Breast Cancer in Women Aged 40 - 49 Years. Journal of Midlife Health, 8, 2-10. https://doi.org/10.4103/jmh.JMH_26_16

[6] Agrawal, A., Tripathi, P., Sahu, A. and Daftary, J. (2014) Breast Screening Revisited. Journal of Family Medicine and Primary Care, 3, 340-344. https://doi.org/10.4103/2249-4863.148103

[7] Singla, D., Chaturvedi, A.K., Aggarwal, A., Rao, S.A., Hazarika, D. and Mahawar, V. (2018) Comparing the Diagnostic Efficacy of Full Field Digital Mammography with Digital Breast Tomosynthesis Using BIRADS Score in a Tertiary Cancer Care Hospital. Indian Journal of Radiology, 28, 115-122. https://doi.org/10.4103/ijri.IJRI_107_17

[8] Choi, E.J., Choi, H., Choi, S.A. and Youk, J.H. (2016) Dynamic Contrast-Enhanced Breast Magnetic Resonance Imaging for the Prediction of Early and Late Recurrences in Breast Cancer. Medicine (Baltimore), 95, e5330. https://doi.org/10.1097/MD.0000000000005330

[9] Elsamaloty, H., Elzawawi, M.S., Mohammad, S. and Herial, N. (2009) Increasing Accuracy of Detection of Breast Cancer with 3 T MRI. American Journal of Roentgenology, 192, 1142-1148. https://doi.org/10.2214/AJR.08.1226

[10] Le-Petross, H.T., Whitman, G.J., Atchley, D.P., Yuan, Y., Gutierrez-Barrera, A., et al. (2011) Effectiveness of Alternating Mammography and Magnetic Resonance Imaging for Screening Women with Deleterious BRCA Mutations at High Risk of Breast Cancer. Cancer, 117, 3900-3907. https://doi.org/10.1002/cncr.25971

[11] Fiaschetti, V., Pistolese, C.A., Perretta, T., Cossu, E., Arganini, C., et al. (2011) 3-5 BI-RADs Microcalcifications: Correlation between MRI and Histological Findings. ISRN Oncology, 2011, Article ID: 643890. 
[12] Dorrius, M.D., Pijnappel, R.M., Weide, M.C. and Oudkerk, M. (2010) Breast Magnetic Resonance Imaging as a Problem-Solving Modality in Mammographic BI-RADS 3 Lesions. Cancer Imaging, 10, S54-S58. https://doi.org/10.1102/1470-7330.2010.9020

[13] Hoffmann, S., Shutler, J., Lobbes, M., Burgeth, B. and Bäse, A.M. (2013) Automated Analysis of Non-Mass-Enhancing Lesions in Breast MRI Based on Morphological, Kinetic, and Spatio-Temporal Moments and Joint Segmentation-Motion Compensation Technique. EURASIP Journal on Advances in Signal Processing, 2013, 172. https://doi.org/10.1186/1687-6180-2013-172

[14] Morris, E. (2006) Breast MR Imaging Lexicon Updated. Magnetic Resonance Imaging Clinics of North America, 14, 293-303. https://doi.org/10.1016/j.mric.2006.07.001

[15] Macura, K., Ouwerkerk, R., Jacobs, M. and Bluemke, D.A. (2006) Patterns of Enhancement on Breast MR Images: Interpretation and Imaging Pitfalls. RadioGraphics, 26, 1719-1734. https://doi.org/10.1148/rg.266065025

[16] Kuhl, C. (2006) Concepts for Differential Diagnosis in Breast MR Imaging. Magnetic Resonance Imaging Clinics, 14, 305-328.

[17] Turnbull, L., Brown, S., Harvey, I., Olivier, C., Drew, P., et al. (2010) Comparative Effectiveness of MRI in Breast Cancer (COMICE) Trial: A Randomised Controlled Trial. The Lancet, 375, 563-571. https://doi.org/10.1016/S0140-6736(09)62070-5

[18] Tozaki, M., Igarashi, T. and Fukuda, K. (2006) Positive and Negative Predictive Values of BI-RADS Descriptors for Focal Breast Masses. Magnetic Resonance in Medical Sciences, 5, 7-15. https://doi.org/10.2463/mrms.5.7

[19] Kul, S., Cansu, A., Alhan, E., Dinc, H., Gunes, G. and Reis, A. (2011) Contribution of Diffusion-Weighted Imaging to Dynamic Contrast-Enhanced MRI in the Characterization of Breast Tumors. American Journal of Roentgenology, 196, 210-217. https://doi.org/10.2214/AJR.10.4258

[20] Rubesova, E., Grell, A.S. and Maertelaer, D.V. (2006) Quantitative Diffusion Imaging in Breast Cancer: A Clinical Prospective Study. Journal of Magnetic Resonance Imaging, 24, 319-324. https://doi.org/10.1002/jmri.20643

[21] Imamura, T., Isomoto, I., Sueyoshi, E., et al. (2010) Diagnostic Performance of ADC for Non Mass Like Breast Lesions on MR Imaging. Magnetic Resonance in Medical Sciences, 9, 217-225. https://doi.org/10.2463/mrms.9.217

[22] Yabuuchi, H., Matsuo, Y., Kamitani, T., Setoguchi, Okafuji, T., Soeda, H., et al. (2010) Non-Mass-Like Enhancement on Contrast-Enhanced Breast MR Imaging: Lesion Characterization Using Combination of Dynamic Contrast-Enhanced and Diffusion-Weighted MR Images. European Journal of Radiology, 75, 126-132.

[23] Palle, L. and Reddy, B. (2009) Role of Diffusion MRI in Characterizing Benign and Malignant Breast Lesions. The Indian Journal of Radiology and Imaging, 19, 287-290. https://doi.org/10.4103/0971-3026.57209 